
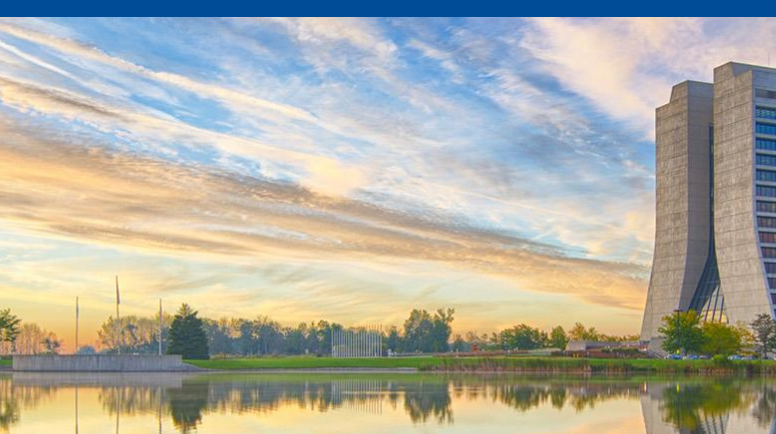

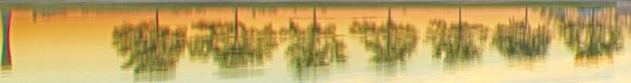

\title{
A multi-channel cryogenic low-noise skipper-CCD readout ASIC
}

FERMILAB-SLIDES-21-113-QIS

Troy England ${ }^{1}$, Fabricio Alcalde Bessia ${ }^{2,3}$, Hongzhi Sun ${ }^{1}$, Leandro Stefanazzi ${ }^{1}$, Davide Braga ${ }^{1}$, Miguel Sofo Haro 2,4 , Shaorui Li ${ }^{1}$, Juan Estrada1', Farah Fahim¹

${ }^{1}$ Fermi National Accelerator Laboratory, Batavia, Illinois, USA

${ }^{2}$ Consejo Nacional de Investigaciones Científicas y Técnicas (CONICET), Buenos Aires, Argentina

${ }^{3}$ Instituto Balseiro, San Carlos de Bariloche, Argentina

${ }^{4}$ Centro Atómico Bariloche, Instituto Balseiro, Comisión Nacional de Energía Atómica (CNEA), Argentina 


\section{Talk Abstract}

- Skipper CCDs provide exceptionally low noise useful for dark matter detection

- MIDNA ASIC enables scaled cryogenic Skipper CCD readout

- Noise performance proven to specification at room temperature with CCD

- Liquid nitrogen measurements show functionality with more to come

- Future development will integrate more sensitive signals and averaging capability 


\section{Skipper CCDs for Exceptionally Low Noise}

- Skipper CCDs and similar technologies enable the non-destructive readout of the same pixel many times [1-4]

- The noise can be lowered by averaging many shorter reads rather than one long read

- It continues the decreasing noise trend with longer measurement times otherwise limited by $1 / f$ noise

- There are still limits to this approach

- Leakage in the charge transport in the CCD (low)

- Speed of the various readout stages and clocking

- A skipper CCD has demonstrated the lowest ever, $0.068 \mathrm{e}_{r \mathrm{rss}}^{-}$, noise floor in CCDs [3]
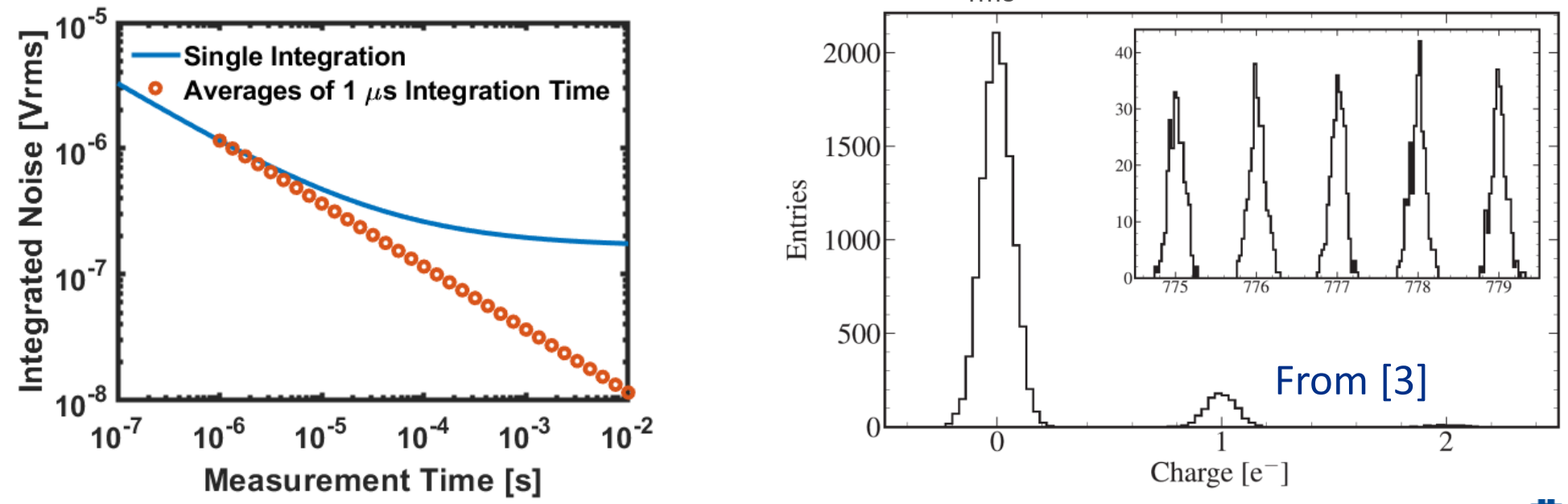

[1] White et al., "Characterization of Surface Channel CCD Image Arrays at Low Light Levels." 


\section{OSCURA 4 year Research and Development Effort}

“DOE Dark matter New Initiatives" FNAL, LBNL, PNNL, U. Chicago, U. Washington, Stony Brook University.

Fermilab is leading the effort to develop a skipper-CCD dark matter detector with active mass of $10 \mathrm{~kg}$ of Silicon.

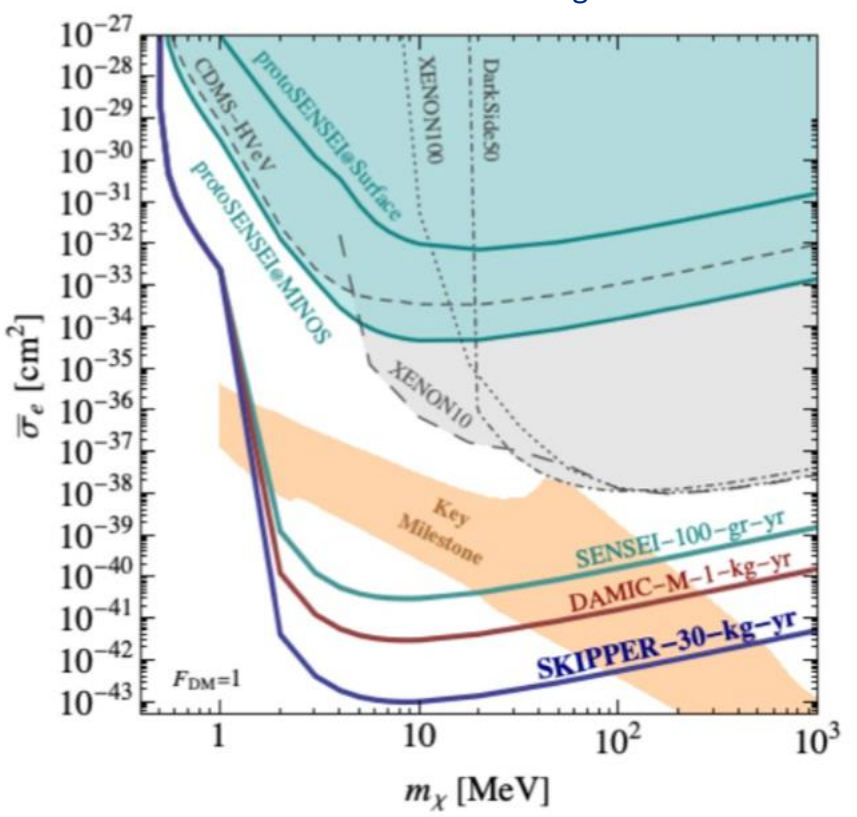

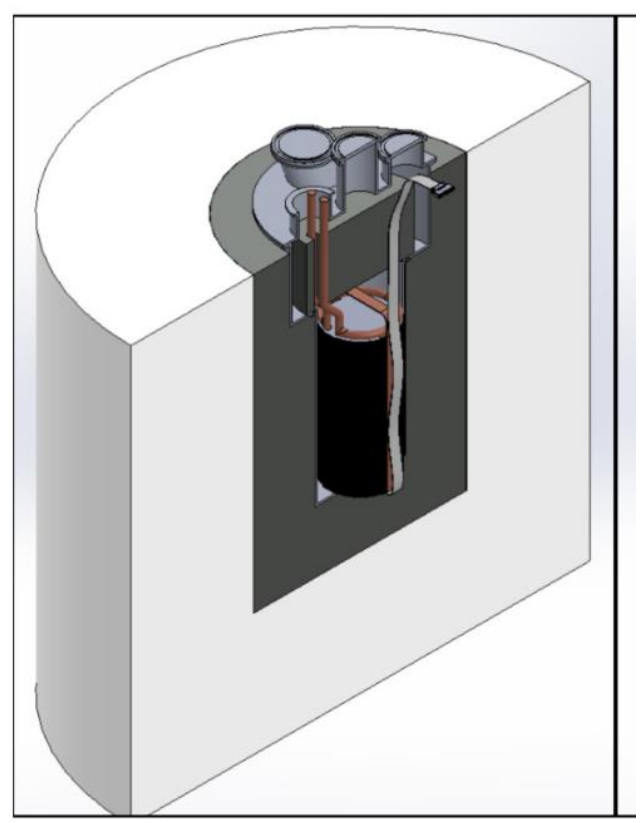

\section{Gigapixel digital camera for} dark matter!

Cooling, readout, packaging and testing of the required 4000 skipper-CCD sensors require engineering solutions that are not available yet for scientific CCDs.

Sensors designed by Steve Holland at LBNL.

Radiation background required is $\approx 10 x$ lower than state of the art experiments.

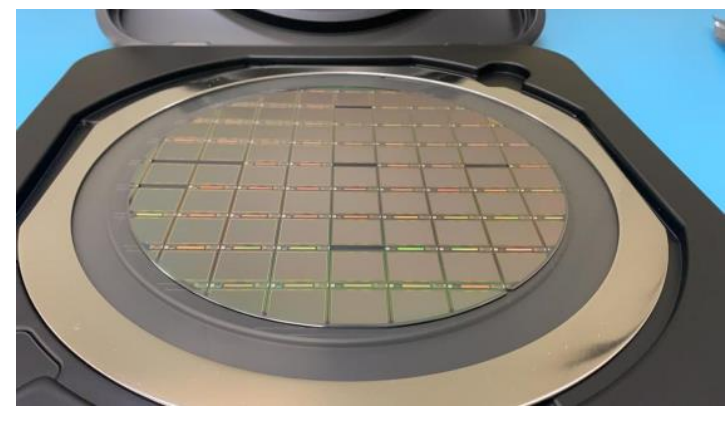

Fabrication of skipper-CCDs is being adapted to the changes in the semiconductor industry. We have new CCDs from Microchip and are testing the fabrication results.

Taking the skipper-CCDs to their full potential as dark matter detectors. 


\section{An ASIC to Support Scaling to 28 Gigapixels, MIDNA}

- MIDNA is a prototype cryogenic low-noise skipper-CCD readout ASIC

- It is an enabling technology for the OSCURA dark matter detection project

- Integrates multiple readout channels onto a single chip, replacing numerous costly PCB components and saving valuable physical space

- 4 channels on prototype chip

- Uses $2.5 \mathrm{~V}$ transistors for voltage room in $65 \mathrm{~nm}$ CMOS process

- Operates at 120 Kelvin for optimum CCD performance

- COTS devices not guaranteed to specification

- The ASIC input referred noise must be less than one third of the CCD output noise

- Most difficult and constraining requirement
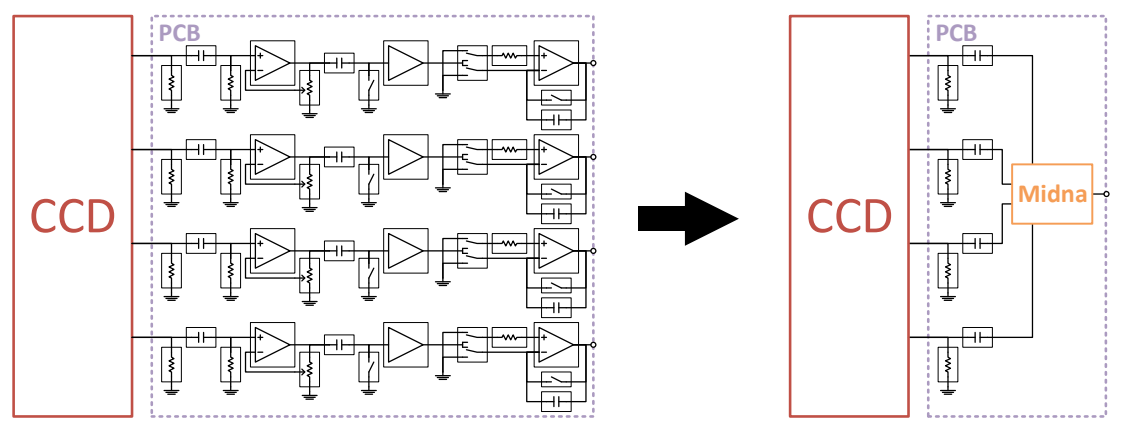


\section{MIDNA Signal Chain}

- Preamplifier largely responsible for noise performance drives power consumption

- DC Restore prevents the saturation of the integrator sticking baseline to $V_{R E F}$

- Buffer to provide the current necessary for the Integrator

- Switchable Integrator to implement CDS on chip with efficient bandwidth

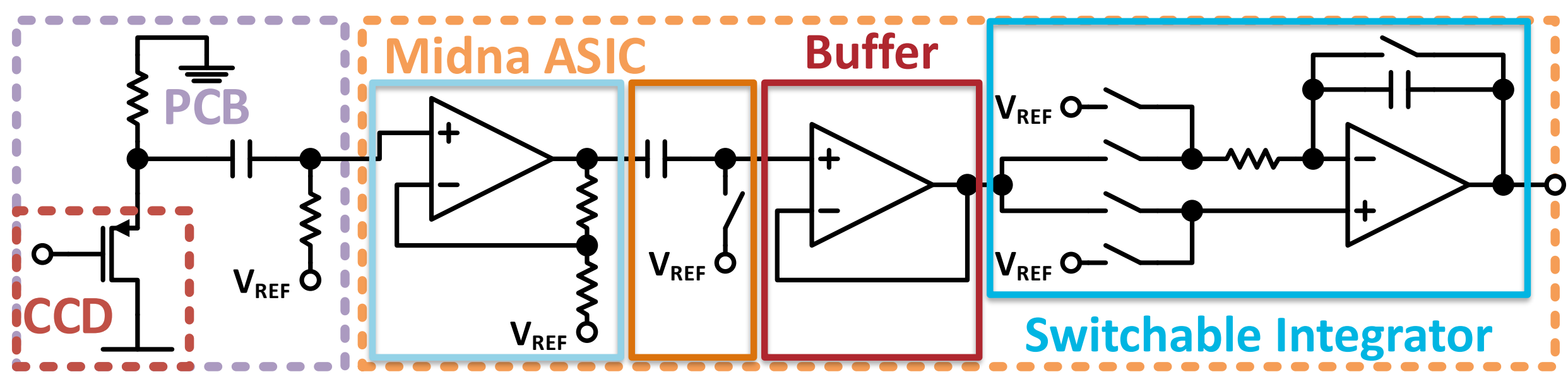

Preamp DC Restore 


\section{Chip Image and Tape Out Structures}

- Each channel is about $150 \mu \mathrm{m} \times 1 \mathrm{~mm}$; Chip is $2 \mathrm{~mm} \times 1 \mathrm{~mm}$

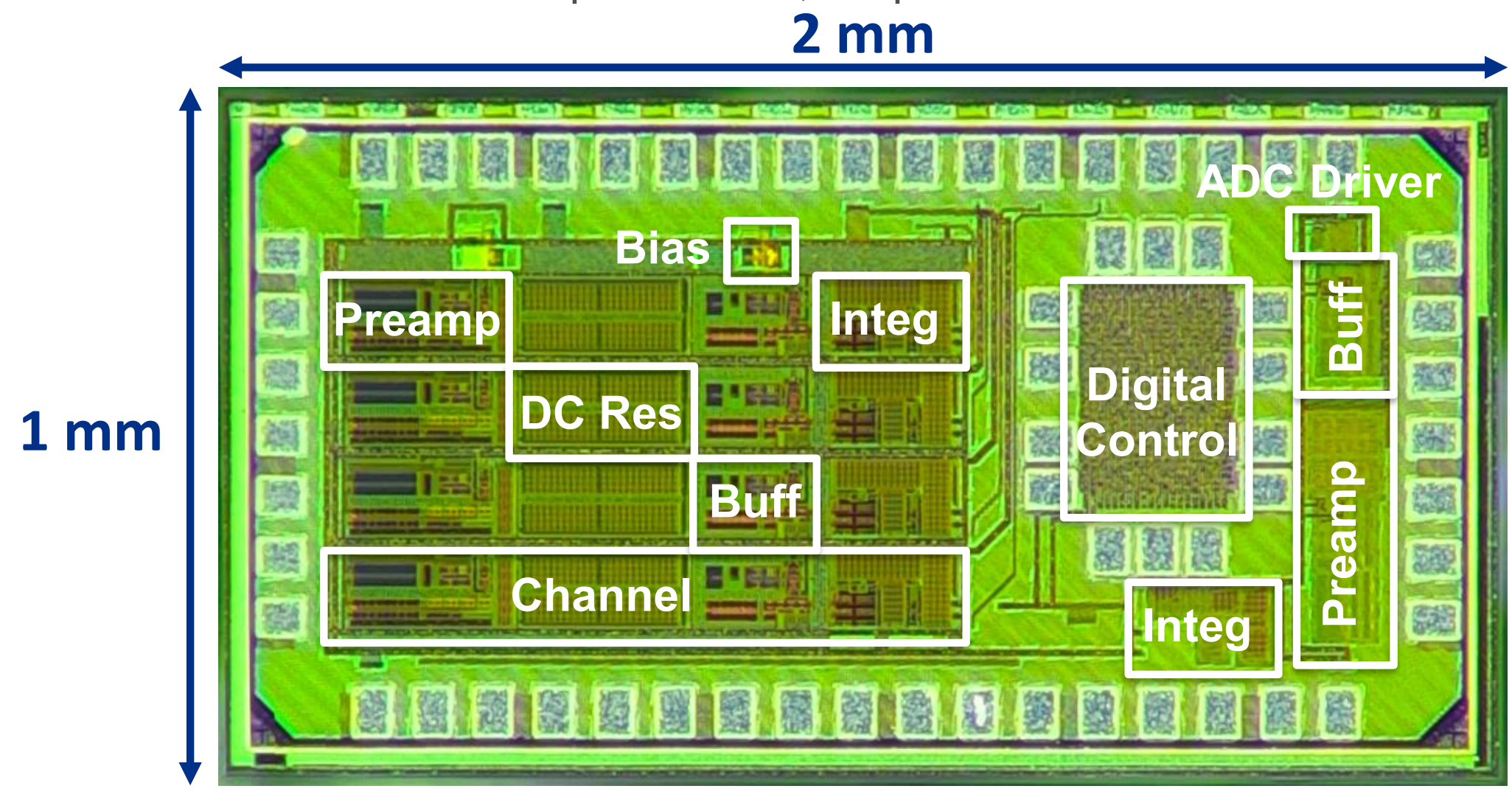




\section{Room Temperature MIDNA with Cooled CCD Testing Setup}

DAQ board and MIDNA at room temperature
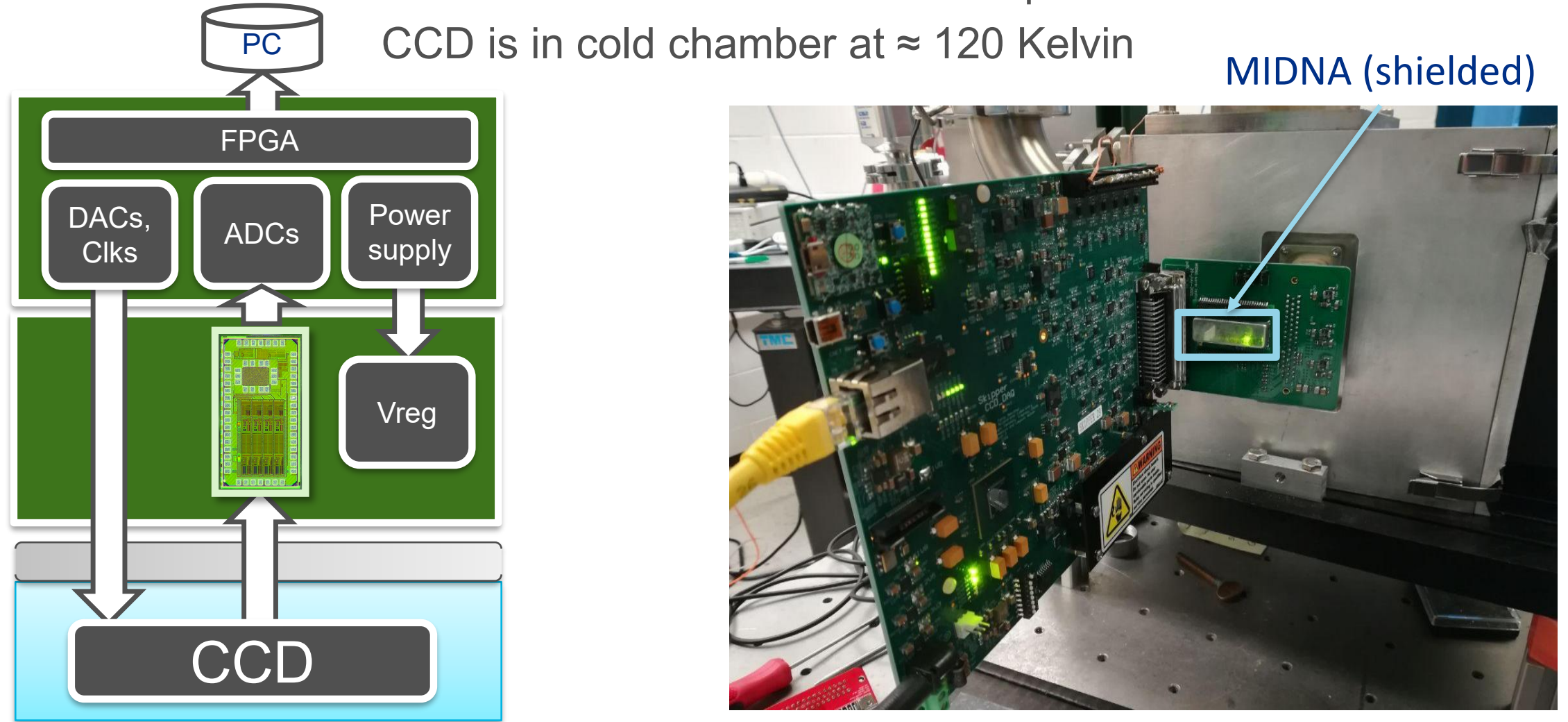


\section{Room Temp. MIDNA and Cooled CCD Gain Measurements}

- Fe-55 X-ray source $\rightarrow 5.9 \mathrm{keV}$ photons $\rightarrow 1639$ e- on average

- Histogram of single photon hits
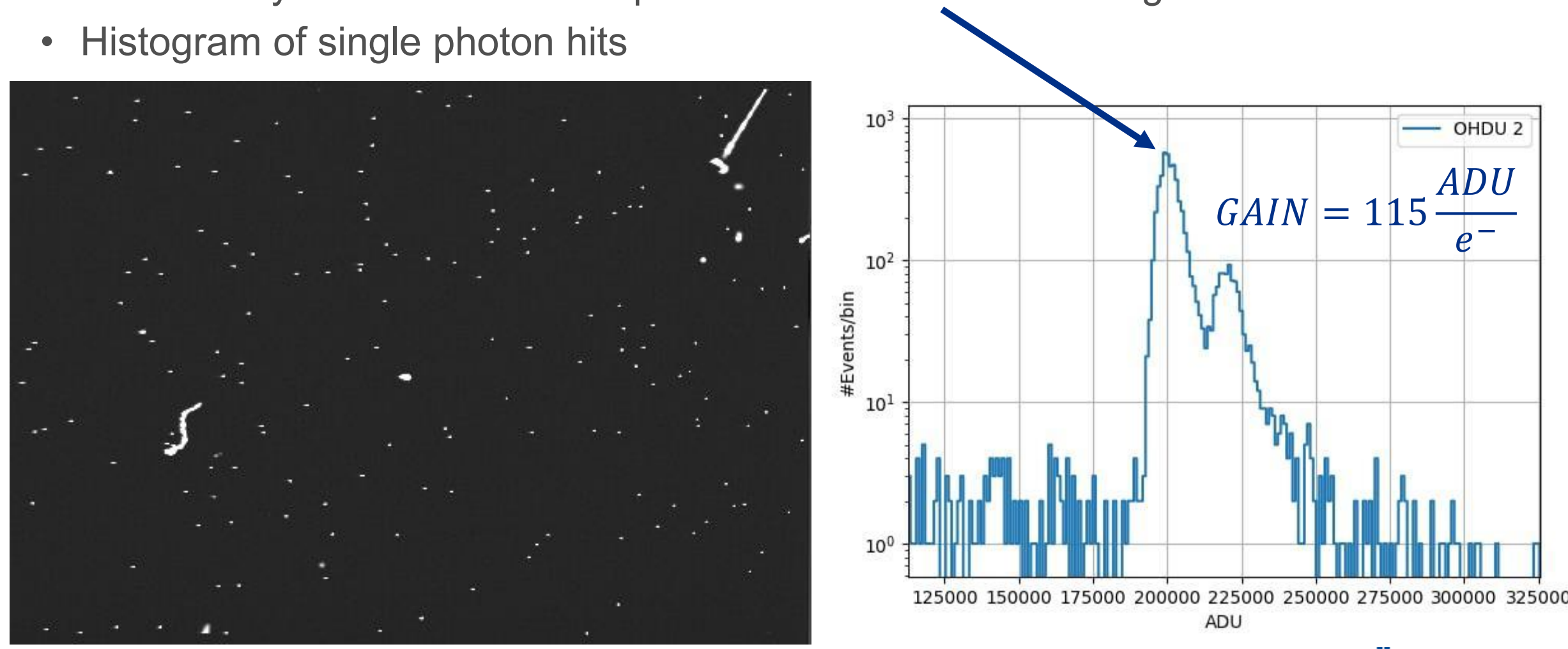


\section{Room Temp. MIDNA and Cooled CCD Noise Measurements}

- Measured as the standard deviation on the overscan pixels, region of no charge

- 10 us integration time in each direction

- Noise dominated by CCD

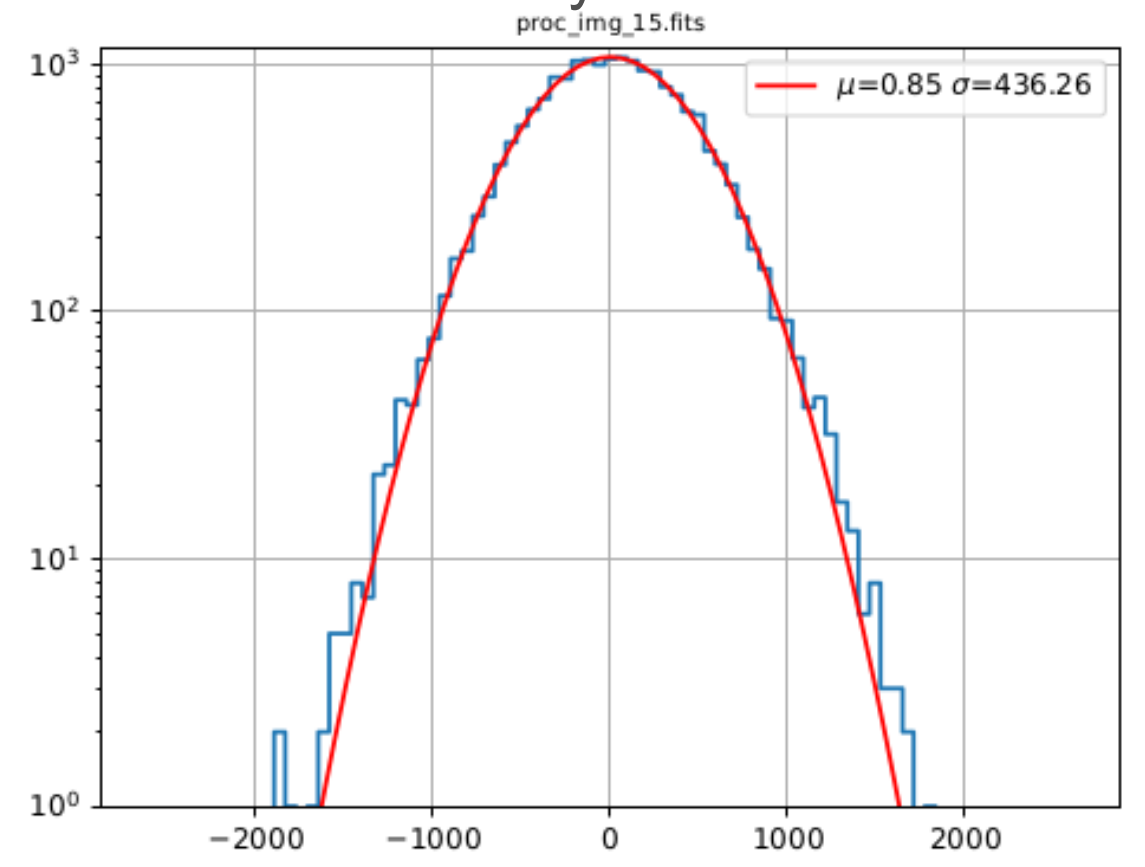

$$
N O I S E=\frac{S T D}{G A I N}=\frac{[A D U]}{\left[\frac{A D U}{e^{-}}\right]}
$$

\begin{tabular}{|l|l|}
\hline & Noise \\
\hline Simulated & $3.10 \mathrm{e}_{\mathrm{rms}}^{-}$ \\
\hline Measured & $3.79 \mathrm{e}_{\mathrm{rms}}^{-}$
\end{tabular}

Matches performance of discrete solution with smaller footprint and at one tenth of the power! 


\section{Cooled MIDNA Functionality}

- Functionality proven in liquid nitrogen!

- Measurements inside of temperature controlled dewar are still in progress
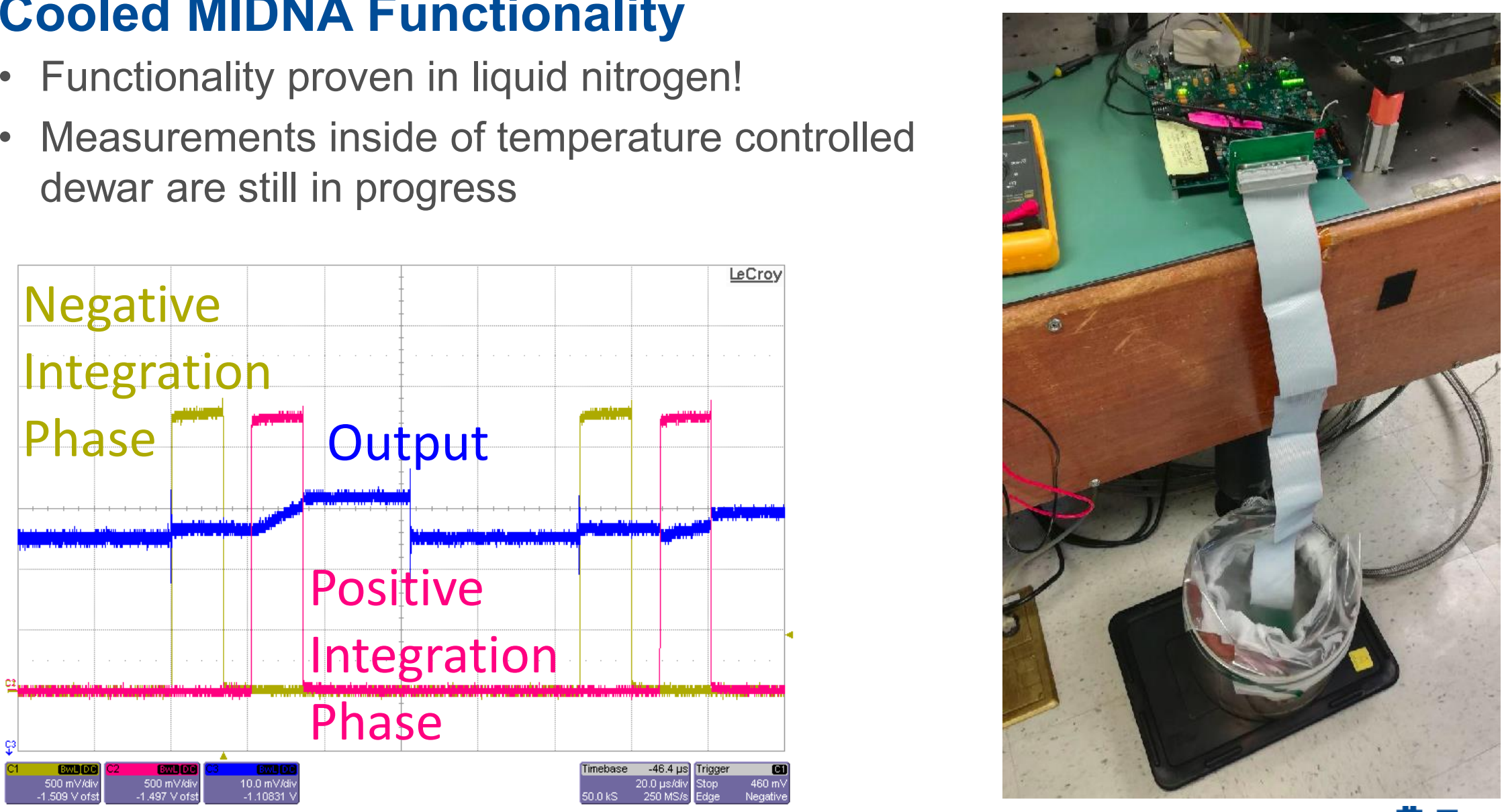


\section{Noise Measurements at Room Temperature and Liquid Nitrogen}

- At room temperature, single integration noise with MIDNA alone is successfully $1 / 3$ of noise with CCD included!

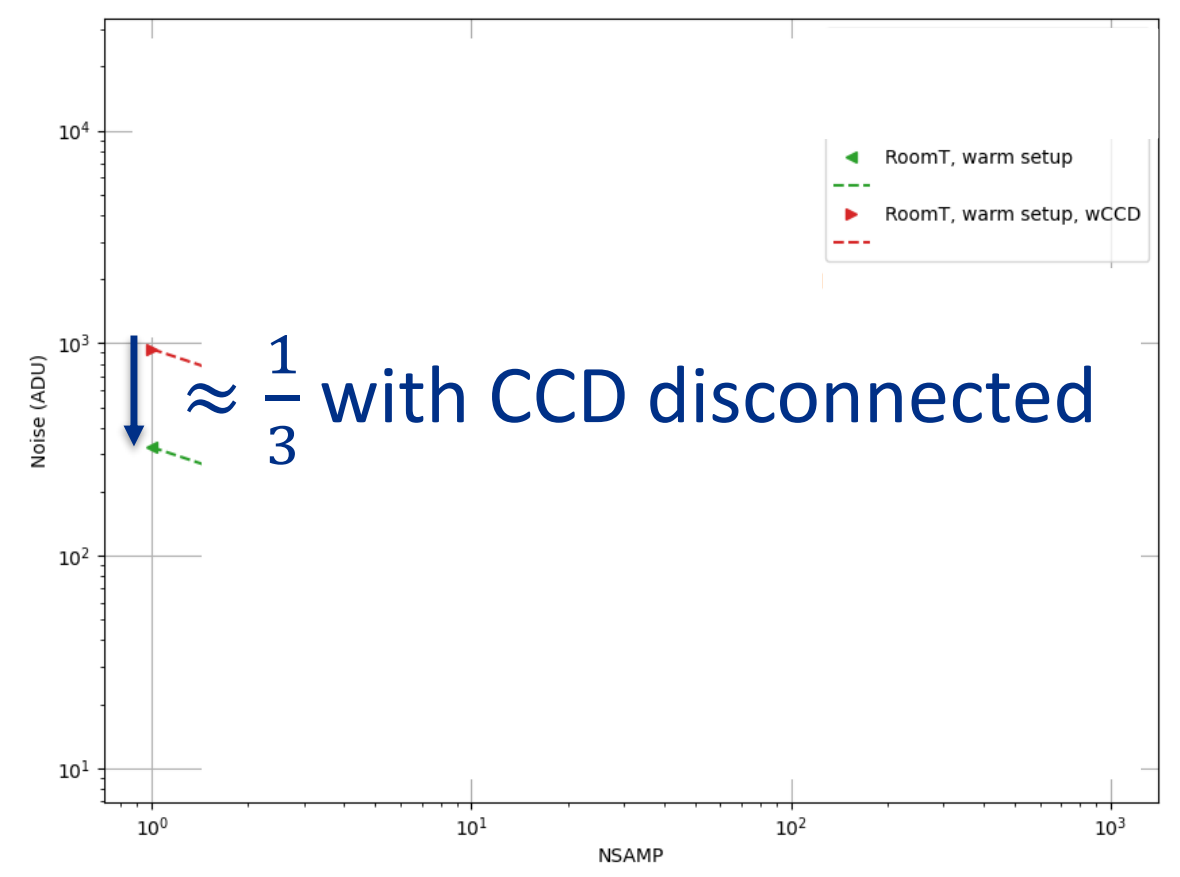




\section{Noise Measurements at Room Temperature and Liquid Nitrogen}

- At room temperature, single integration noise with MIDNA alone is successfully $1 / 3$ of noise with CCD included!

- Averaging saturates out likely due to 1/f noise being injected after CDS

- Mitigations are being investigated

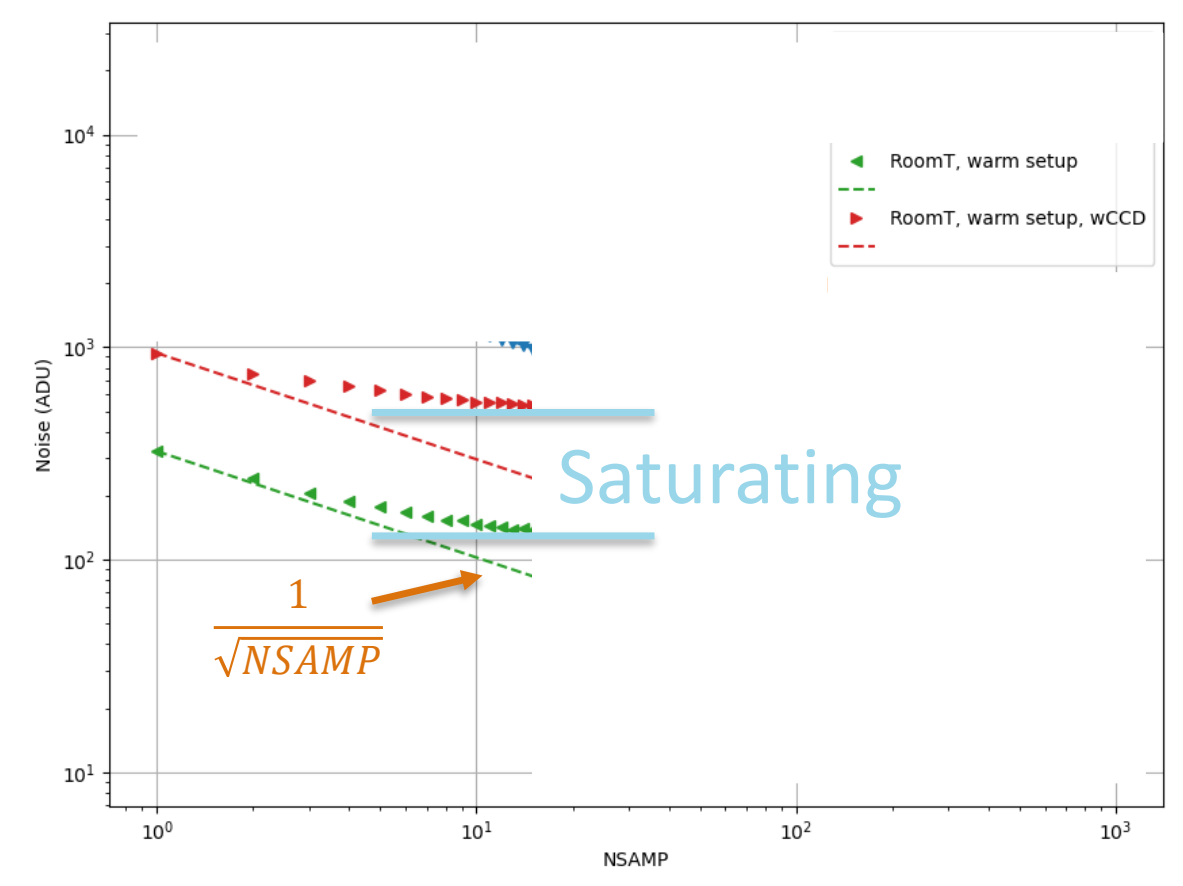




\section{Noise Measurements at Room Temperature and Liquid Nitrogen}

- At room temperature, single integration noise with MIDNA alone is successfully $1 / 3$ of noise with CCD included!

- Averaging saturates out likely due to 1/f noise being injected after CDS

- Mitigations are being investigated

- Liquid nitrogen setup requires long ribbon cabling that is coupling in noise even without dunking

- Dunking continues to make it worse

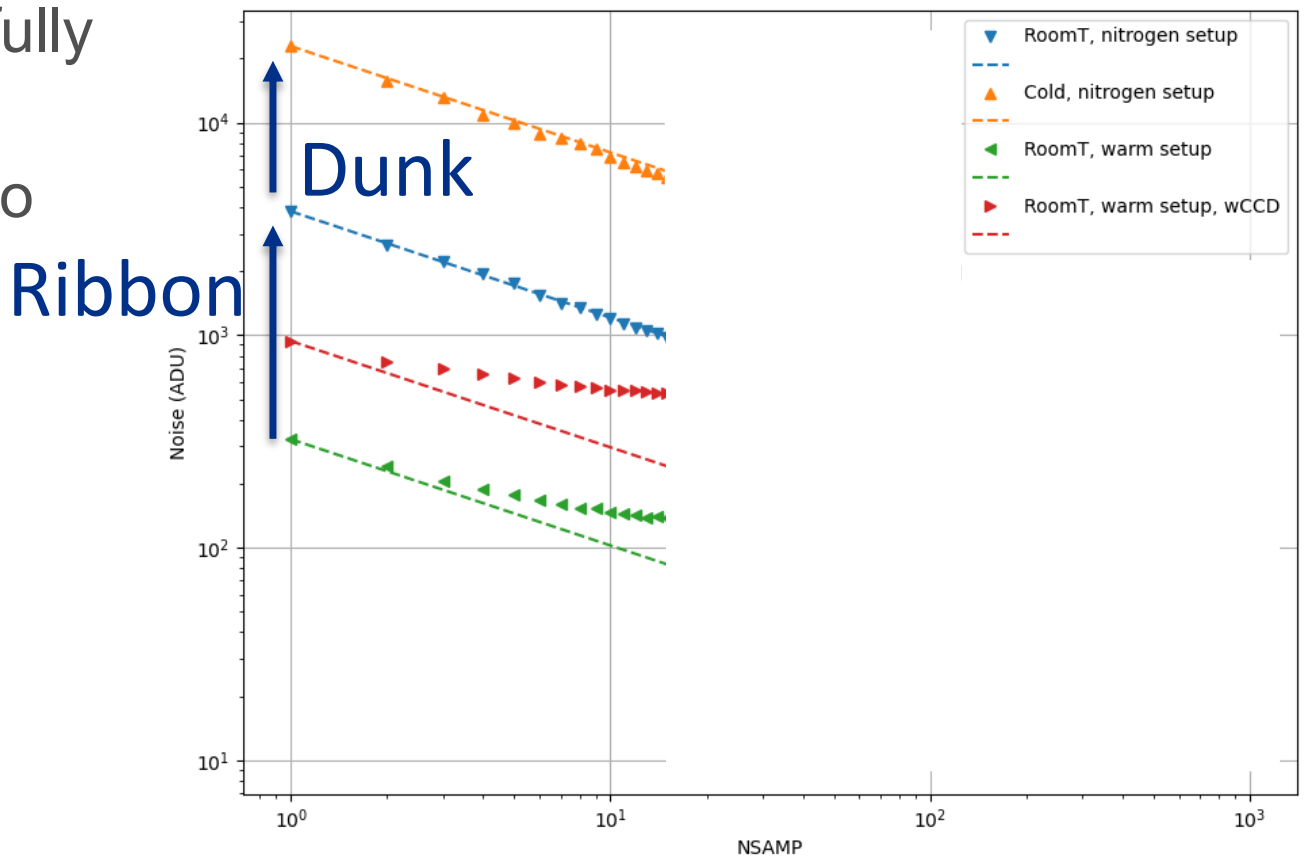




\section{Noise Measurements at Room Temperature and Liquid Nitrogen}

- At room temperature, single integration noise with MIDNA alone is successfully $1 / 3$ of noise with CCD included!

- Averaging saturates out likely due to 1/f noise being injected after CDS

- Mitigations are being investigated

- Liquid nitrogen setup requires long ribbon cabling that is coupling in noise even without dunking

- Dunking continues to make it worse

- Follows $\frac{1}{\sqrt{\text { NSAMP }}}$ much better while

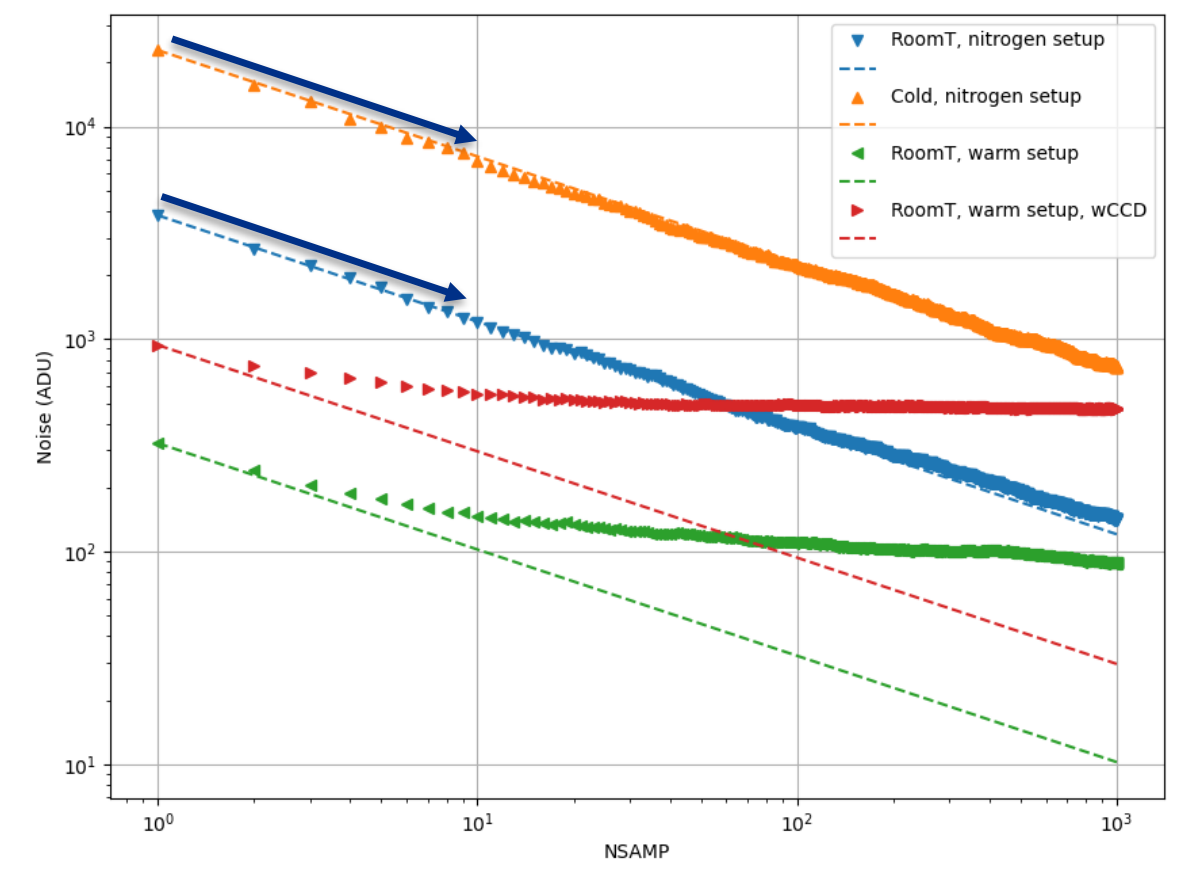
starting much higher

- More work to come 


\section{Results and Future Work for MIDNA}

- Successfully less than 1/3 of CCD noise even at room temperature!

- Cryogenic functionality down at 77 Kelvin!

- Controlled temperature testing with CCD in progress

- Starting design work for next version of chip

- Grow to many more channels to follow the CCD, up to 64

- Project scaling is currently limited by cost of readout

MIDNA Chip Image

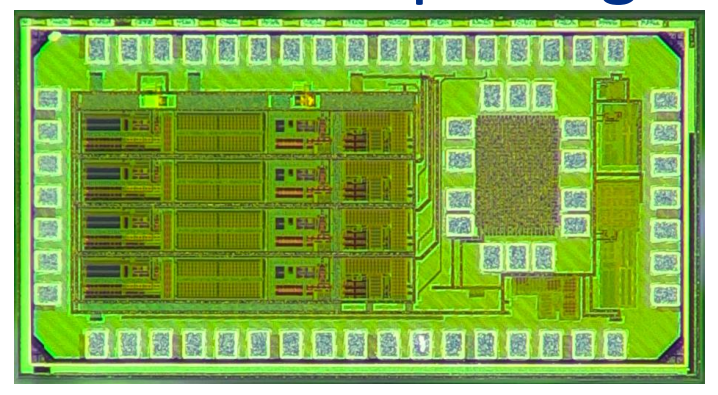

- Integrate reference generator and analog-to-digital converter

- Greatly reduce the sensitive analog signals going off chip

- Enable digital processing of data on chip

- The ADC combined with on-chip memory enables many averaged reads internally

- Improves noise, power, and complexity compared to analog solutions, especially off chip 


\section{Thanks!}

